\title{
Molecular identification of Giardia duodenalis isolates from domestic dogs and cats in Wroclaw, Poland
}

\author{
Jolanta Piekarska', Joanna Bajzert² , Michał Gorczykowski', Magdalena Kantyka', \\ Magdalena Podkowik ${ }^{3}$

\begin{abstract}
${ }^{1}$ Department of Internal Medicine and Clinic of Diseases of Horses, Dogs and Cats, Division of Parasitology, Faculty of Veterinary Medicine, Wroclaw University of Environmental and Life Sciences, Poland

2 Department of Immunology, Pathophysiology and Veterinary Preventive Medicine, Division of Immunology and Veterinary Preventive Medicine, Faculty of Veterinary Medicine, Wroclaw University of Environmental and Life Sciences, Poland ${ }^{3}$ Department of Food Hygiene and Consumer Health Protection, Division of Food Microbiology and Processing Hygiene, Faculty of Veterinary Medicine, Wroclaw University of Environmental and Life Sciences, Poland
\end{abstract}

\begin{abstract}
Piekarska J, Bajzert J, Gorczykowski M, Kantyka M, Podkowik M. Molecular identification of Giardia duodenalis isolates from domestic dogs
\end{abstract} and cats in Wroclaw, Poland. Ann Agric Environ Med. 2016; 23(3): 410-415. doi: 10.5604/12321966.1219178

\begin{abstract}
Introduction. Giardia duodenalis (G. intestinalis) is a common protozoan causing gastrointestinal disorders in many species of mammals. The genus of Giardia has high molecular diversity. Dogs and cats, in addition to their typical infection with assemblages $C, D$ and $F$, may be a reservoir of zoonotic assemblages ( $A$ and $B$ ).

Objective. The aim of this study was a genetic characteristic of Giardia isolates of dogs and cats from the area of Wroclaw (Poland).

Materials and method. A total of 128 and 33 faecal samples from dogs and cats, respectively, were analyzed by routine coprological methods. The animals were diagnosed on the presence of $G$. duodenalis antigens in faeces soluble with the use of SNAP Giardia (IDEXX Laboratories) immunosorbent assay. 27 DNA isolates of Giardia were subjected to molecular identification (PCR-RFLP).

Results and conclusions. The prevalence of $G$. duodenalis was $21.1 \%(27 / 128)$ in dogs and $15.1 \%(5 / 33)$ in cats. In dogs, C assemblage was present in $18(81 \%)$ positive stool samples, D assemblage in 2 (9\%) samples, B assemblage present in one (4.5\%), and mixed assemblages (C and D) occurred in one (4.5\%) sample. F assemblage was found in 4 (80\%) cats' positive stool samples and A assemblage occurred in one case (20\%). Confirmation of the presence of A and B zoonotic assemblages suggests that infected pets can be a threat to human health. This study describes for the first time the presence of mixed infections within host-specific $C$ and $D$ assemblages in dogs in Poland.
\end{abstract}

\section{Key words}

Giardia duodenalis, assemblage, dogs, cats, nested-PCR, PCR-RFLP, zoonosis

\section{INTRODUCTION}

Giardia duodenalis (G. intestinalis, G. lamblia) is a widespread protozoan parasitizing in humans and many species of mammals. Invasion is most commonly associated with the occurrence of gastrointestinal signs, though asymptomatic invasions have also been observed [1]. Studies using molecular techniques have shown that genetic diversity is very high within the $G$. duodenalis species. There are 7 basic assemblages, from A - G. The occurrence of A, B, C and $\mathrm{D}$ assemblages was confirmed in dogs, and $\mathrm{A}, \mathrm{B}, \mathrm{D}$ and $\mathrm{F}$ assemblages found in cats. Due to the occurrence of $A$ and $B$ assemblages in humans, and because of their relevance for zoonotic infections, there is a need for the monitoring of dogs and cats as companion animals that can be a direct source of human infection, as well as a source of environmental contamination $[2,3]$. Asymptomatic and chronic course of the disease with frequent periodic expulsion of cysts is

Address for correspondence: Jolanta Piekarska, Department of Parasitology, Faculty of Veterinary Medicine, Wroclaw University of Environmental and Life Sciences, Poland

E-mail: jolanta.piekarska@up.wroc.pl

Received: 19 October 2013; accepted: 01 July 2014 observed in older animals. Diagnosis of Giardia infection with the use of a faecal flotation or faecal smear is problematic due to the irregular shedding of cysts and their morphology, small size, and their similarity in appearance to many pseudoparasites such as yeast [4]. The use of immunoenzyme assay to detect Giardia coproantigens in faeces increases the probability of detecting invasion. Genotypic characterization of $G$. duodenalis is a very useful and essential tool used in epidemiological studies. PCR techniques for genotyping of G. duodenalis are based on polymorphic genes encoding $18 \mathrm{~S}$ rRNA, glutamate dehydrogenase (gdh), triose phosphate isomerase (tpi), and B-giardin [5, 6]. PCR amplification and RFLP/sequence analysis of all of these genes, with the exception of the 18S rRNA, can differentiate subgenotypes of assemblage A [7]. PCR methods for detection of gdh can provide information on $G$. duodenalis A and B subassemblages [8].

\section{OBJECTIVE}

The aim of the study was the genetic characterization of isolates of Giardia in dogs and cats in the area of Wroclaw (Poland), which is important in determining the source of 
the invasion in humans and animals in a given area, as well as in assessment of the zoonotic potential of $G$. duodenalis.

\section{MATERIALS AND METHOD}

Samples collection and qualification. Faecal samples of dogs and cats (age: 3 weeks - 10 years) from Wroclaw were collected in 2010-2012 and provided by their owners to the Division of Parasitology, Faculty of Veterinary Medicine (Wroclaw). The total number of stool samples was 161 (128 dogs and 33 cats), all of the animals had different gastric symptoms (e.g diarrhea, emaciation, loss of body weight). Faecal samples were stored at $-20^{\circ} \mathrm{C}$. The animals were diagnosed on the presence of G. duodenalis antigens in faeces soluble with the use of SNAP Giardia (IDEXX Laboratories) immunosorbent assay. As a result of such qualifications, material for genetic research was obtained from 32 animals (27 dogs and 5 cats). Pets with positive test results ranged in age from 3 weeks -2 years.

DNA isolation. Faecal samples (approximately 3-4 g) from infected animals were examined by the flotation method with the use of saturated $\mathrm{NaCl}$ solution. The upper part of the supernatant was harvested, rinsed in distilled $\mathrm{H}_{2} \mathrm{O}$, centrifuged at 1,000 rpm for 5 minutes. The resulting pellet was re-suspended in $300 \mu$ l of saline. This was a concentrated sample of Giardia cysts, which were the basis for DNA isolation using a Genomic Mini AX STOOL kit (DNAGdansk, Poland).

Genotyping and nested PCR. Giardia genotyping was performed based on the polymorphism of a gene fragment coding for $\beta$-giardin. Nested PCR technique for DNA amplification was applied. Reaction parameters and primers sequences were described in Lalle et al. (2005) [9]. In the primary PCR reaction forward G7: 5'AAGCCCGACGACCTCACCCGCAGTGC3' and reverse G759: 5'GAGGCCGCCCTGGATCTTCGAGACGAC3' primers were used. The reaction mixture consisted of $400 \mathrm{nM}$ of each primer, $1 \mathrm{x}$ reaction buffer for polymerase DNA Delta3, $500 \mu \mathrm{M}$ of dNTP, $3 \mathrm{mM}$ of $\mathrm{MgCl}_{2}, 1.25 \mathrm{U}(0,05 \mathrm{U} / \mu \mathrm{l})$ of polymerase DNA Delta3 (DNA-Gdansk, Poland) and $0.5 \mu \mathrm{l}$ of DNA in a final volume of $25 \mu \mathrm{l}$. The amplification was carried out in a MJ Mini thermal cycler (Bio-Rad) using the following conditions: cycle of $96^{\circ} \mathrm{C}$ for $5 \mathrm{~min} ; 5$ cycles of: initially denaturation for $30 \mathrm{sec}$ at $95^{\circ} \mathrm{C}$, annealing for $30 \mathrm{sec}$ at $55^{\circ} \mathrm{C}$, polymerization for $1 \mathrm{~min}$ at $72^{\circ} \mathrm{C}$. The PCR cycle was then carried out for $30 \mathrm{sec}$ at $95^{\circ} \mathrm{C}, 30 \mathrm{sec}$ at $65^{\circ} \mathrm{C}$ and $1 \mathrm{~min}$ at $72^{\circ} \mathrm{C}$, for a total of 30 cycles, followed by a final extension for $10 \mathrm{~min}$ at $72^{\circ} \mathrm{C}$. The secondary PCR reaction was amplified with forward 511: 5' GAACGAACGAGATCGAGGTCCG'3 and reverse 511: 5' CTCGACGAGCTTCGTGTT 3' primers. The amplification conditions were almost the same as in the first reaction with only 2 differences. The annealing temperature was changed, the first was $50^{\circ} \mathrm{C}$ and the second was $55^{\circ} \mathrm{C}$ and no final extension was carried out. The amplicons of the first PCR reaction (753 bp) was the matrix of secondary PCR reaction.

Amplicons of second PCR reaction (total volume $=100 \mu \mathrm{l}$ ) were subjected to electrophoresis in $2 \%$ agar gel. PCR products with a length of $\sim 511 \mathrm{bp}$ were isolated from the gel and purified using a set of DNA Extraction Kit (Fermentas). DNA concentration was determined by absorbance measurements at $260 \mathrm{~nm}$ wavelength. Purified products were digested with restriction enzyme BsuRI/HaeIII (Fermentas) for 2 hours at $37^{\circ} \mathrm{C}$. The required enzyme amount was calculated based on its activity on the lambda phage. Digestion products were electrophoresed in a $5 \%$ agarose gel for $2 \mathrm{~h}$ at a constant $120 \mathrm{~V}$. Products were stained with ethidium bromide and photographed with a BioRad GelDoxXR device.

Identification of genotypes of sequenced DNA. Selected products of PCR reaction of approximately 511bp were subjected to DNA sequencing by GENOMED S.A. (Poland). The obtained results of sequencing were analyzed by comparing the sequence of individual genotypes of G. duodenalis with BLAST NCBI (http://blast.ncbi.nlm. nih.gov) database. The partial sequences of $\beta$ - giardin gene obtained in this study were compared with sequences deposited in the GenBank database under Accession Nos.: Assemblage A- FJ560591.1; Assemblage B- AY072725.1; Assemblage C- AY545646; Assemblage D- AY545647.1; Assemblage F- AY647264. BioEdit and APE A Plasmid Editor software tools were used for verification of sequence compliance.

\section{RESULTS}

In the study by nested PCR, Giardia DNA was detected in 27 of 32 tested stool samples ( 22 dogs and 5 cats). In 4 cats, the presence of assemblage $F$ was revealed and in 1 cat it was assemblage A. Of the 22 dogs' stool samples, assemblage C was detected in 18 cases, assemblage $\mathrm{D}$ in 2 cases, assemblage $B$ in 1 case.

Selected products of nested PCR reaction ( $\sim 511 \mathrm{bp})$ were sequenced and analyzed using the GenBank database. Analysis of the sequence results in cases where one assemblage was identified clearly confirmed the results obtained from RFLP. Figure 1 shows selected results of sequencing which, in turn, helped to identify the assemblages: A (Fig. 1A), B (Fig. 1B), C (Fig. 1C), D (Fig. 1D), F (Fig. 1E). There were no specific products for any of the previously identified assemblages in electrophoresis of 1 sample derived from a dog after digestion with HaeIII enzyme. The sense strand sequencing results of a $\beta$-gardin gene indicated the presence of assemblage $C$ (Fig. 2A). However, sequencing of the antisense strand showed the presence of assemblage $\mathrm{C}$ or $\mathrm{D}$, giving an appropriate sequence homology at the level of 0.988 and 0.949 . In the analyzed antisense strand stool sample, the cut places were present at 194, 296, 311bp, while characteristic cut places for assemblage $C$ were present at 194 , 296, 311 and 461bp (Fig. 2B) and for assemblage D only in 194 and 311 bp (Fig. 2C). Therefore, it is presumed that in this stool sample from dog we are dealing with a mixed infection of assemblages $\mathrm{C}$ and $\mathrm{D}$, this observation was confirmed by the results of electrophoretic analysis of nested PCR product digested by BsuRI enzyme (data not shown).

In 5 dogs, despite the positive test of SNAP Giardia (IDEXX Laboratories), the PCR product was not detected. The nested PCR repeated one more time with the matrix volume increased to $2-4 \mu \mathrm{l}$ in $25 \mu \mathrm{l}$ of reaction mixture and with the revised polymerase, did not produce satisfactory results. Re-isolation of DNA from thawed samples also did not allow DNA product to be obtained. 
Figure 1 A-E. Multiple alignment of the G. duodenalis assemblage of $\beta$-giardin genes in nested PCR. Alignment based on maximum similarity of individual assemblages of G. duodenalis in the classification of Lalle et al. 2005. The underlined zone indicates a cut place of restrictive enzyme. The grey zone indicates differences in DNA sequence (no consensus at this position)

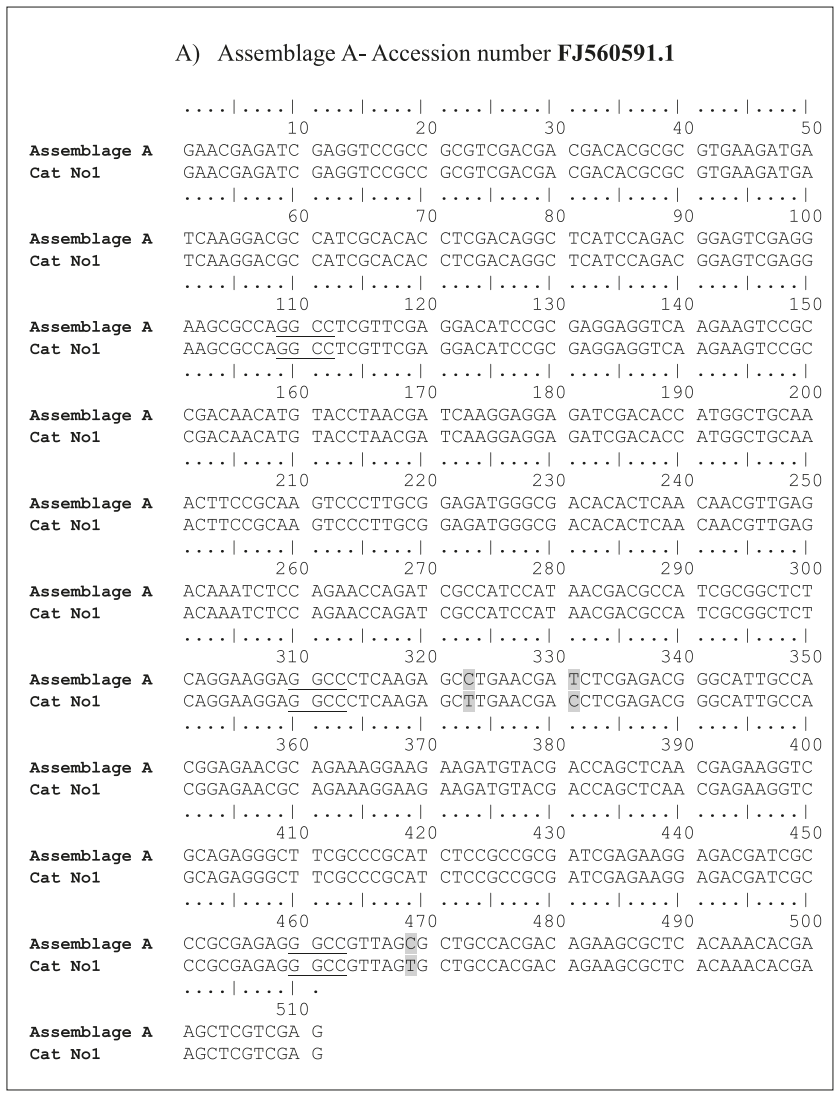

\section{B) Assemblage B- Accession number AY072725.1}

\section{$\ldots|\ldots| \ldots|\cdots|, \ldots|\ldots|, \ldots|\ldots|$} Dog No1 GAACGAGATC GAGGTCCGCC GCGTCGAGGA CGACACGCGT GTGAAGATGA $\ldots|\ldots| \ldots|\ldots| \ldots|\ldots| \ldots|\ldots| \ldots|\ldots| \ldots \mid$ $\begin{array}{rrrrr}60 & 70 & 80 & 90 & 100\end{array}$ Dog No1 TCAAGGACGC CATCGCGCAC CTCGACAGAC TCATCCAGAC AGAGTCGAGG $\ldots|\ldots| \ldots|\ldots| \ldots|\ldots| \ldots|\ldots| \ldots|\ldots| \ldots \mid$ | $\ldots|\ldots|$ Assemblage B AAGCGCCAGG CCTCGTTCGA GGACATCCGC GAGGAAGTCA AGAAGTCTGC Dog No1 AAGCGCCA GG CCTCGTTCGA GGACATCCGC GAGGAGGTCA AGAAGTCTGC

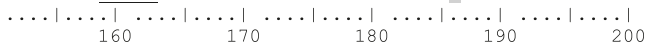
$\begin{array}{cccccc} & 160 & 170 & 180 & 190 & 200 \\ \text { Assemblage B } & \text { CGACAACATG } & \text { TACCTGACGA } & \text { TCAAGGAGA } & \text { GATCGACACT } & \text { ATGGCCGCAA }\end{array}$ Dog no1 CGACAACATG TACCTGACGA TCAAGGAGGA GATCGACACC ATGGCCGCAA

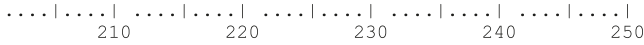

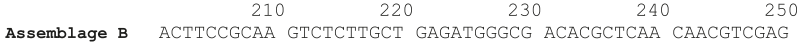
Dog No1 B ACTTCCGCAA GTCTCTTGCT GAGATGGGC ACACGCTCAA CAACGTCGA $\left.\left.\left.\left.\ldots|\ldots|\right|_{260} \ldots|\ldots|{ }_{270} \ldots|\ldots|\right|_{280} \ldots|\ldots|\right|_{290} \ldots|\ldots|\right|_{300}$

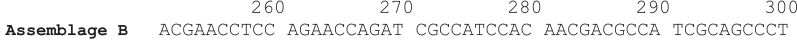
Dog No1 A ACGAACCTCC AGAACCAGAT CGCCATCCAC AACGACGCCA TCGCAGCCC

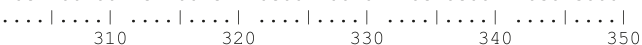
Assemblage B TAGGAAGGAG GCCCTCAAGA GCCTGAACGA CCTCGAGACA GGCATCGCCA Assemblage B
Dog No1

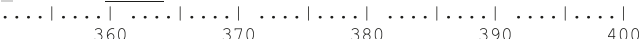
Assemblage B CGGAGAACGC CGAGAGGAag AAGATGTATG ACCAGCTCAA CGAGAAAGTC Dog No1 1 CGGAGAACGC CGAGAGGAAg AAGATGTATG ACCAGCTCAA CGAGAAAGTC

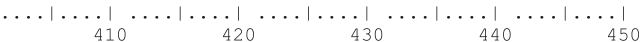
Assemblage B GCAGAGGGCT TCGCCCGCAT CTCCGCTGCC ATCGAGAAGG AGACGATCGC Dog No1 GCAGAGGGCT TCGCCCGCAT CTCCGCTGCC ATCGAGAAGG AGACGATCGC

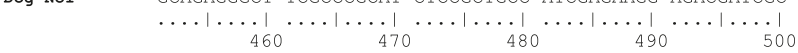
Assemblage B CCGCGAGAGG GCCGTCAGCG CCGCCACGAC AGAGGCCCTC ACAAACACGA Dog No1 CCGCGAGAGG GCCGTCAGCG CCGCCACGAC AGA GGCCCTC ACAAACACGA $\ldots+\ldots 1$

Assemblage B AGCTCGTCGA

Dog No1 AGCTCGTCGA
C) Assemblage C- Accession number AY545646

\begin{tabular}{|c|c|}
\hline & C) Assemblage C-Accession number AY545646 \\
\hline & 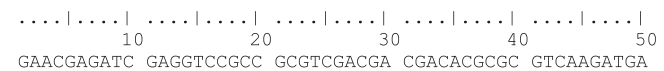 \\
\hline $\begin{array}{l}\text { Assemblage } \mathrm{C} \\
\text { Dog No2 }\end{array}$ & GAACGAGATC GAGGTCCGCC GCGTCGACGA CGACACGCGC GTCAAGATGA \\
\hline & $10{ }_{90} \cdots \cdots+\cdots$ \\
\hline $\begin{array}{l}\text { Assemblage } C \\
\text { Dog No2 }\end{array}$ & 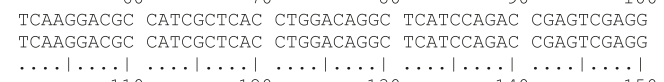 \\
\hline & $120 \quad 130$ \\
\hline Assemblage $\mathrm{C}$ & AAGCGCCAGG GCTCGTTCGA GGACATCCGC GAGGAGGTCA AGAAGTCCGC \\
\hline Dog No2 2 & 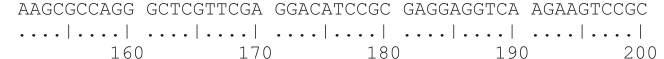 \\
\hline $\begin{array}{l}\text { Assemblage C } \\
\text { Dog No2 }\end{array}$ & $\begin{array}{l}\text { CGACAACATG TACCTGACGA TCAAGGAGGA AATCGACACC ATGGCCGCGA } \\
\text { CGACAACATG TACCTGACGA TCAAGGAGGA AATCGACACC ATGGCCGCGA }\end{array}$ \\
\hline & $\left.\cdots \cdot|\cdots|_{210} \cdots|\cdots|_{220} \cdots|\cdots|\right|_{230} \cdots|\cdots|_{240} \cdots|\cdots|_{250}$ \\
\hline $\begin{array}{l}\text { Assemblage C } \\
\text { Dog No2 }\end{array}$ & $\begin{array}{l}\text { ACTTCCGCAA GTCCCTCGCC GAGATGGGCG AGACCCTCAA CAACGTCGAG } \\
\text { ACTTCCGAA GTCCTCGCC GAGATGGGCG AGACCCTCAA CAACGTCGAG }\end{array}$ \\
\hline & 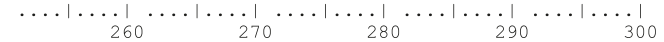 \\
\hline $\begin{array}{l}\text { Assemblage C } \\
\text { Dog No2 }\end{array}$ & 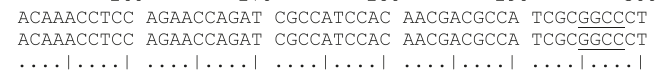 \\
\hline & \\
\hline $\begin{array}{l}\text { Assemblage C } \\
\text { Dog No2 }\end{array}$ & $\begin{array}{l}\text { CAGGAAGGAG GCCCTCAAGA GCCTGACGA CCTCGAGACC GGCATCGCCA } \\
\text { CAGGAAGAGG GCCCTCAAGA GCCTGAACGA CCTCGAGACC GGCATCGCCA } \\
\ldots \ldots|\cdots|\end{array}$ \\
\hline $\begin{array}{l}\text { Assemblage C } \\
\text { Dog No2 }\end{array}$ & $\begin{array}{l}360 \quad 330 \quad 380 \quad 390 \quad 400 \\
\text { CGGAGAACGC CGAGAGGAG AAGATGTACG ACCAGCTCAA CGAGAAGGTC } \\
\text { CGGAGAACGC CGAGAGGAAG AAGATGTACG ACCAGCTCAA CGAGAAGGTC }\end{array}$ \\
\hline & ${ }_{410} \cdots\left|\cdots_{420} \cdots\right| m_{430} \cdots \cdots_{440} \cdots$ \\
\hline $\begin{array}{l}\text { Assemblage } C \\
\text { Dog No2 }\end{array}$ & 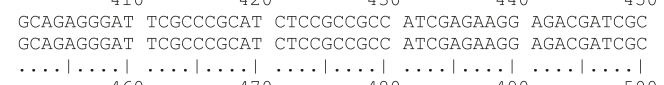 \\
\hline $\begin{array}{l}\text { Assemblage C } \\
\text { Dog No2 }\end{array}$ & $\begin{array}{l}460 \\
\text { CCGCGAGAGG GCCGTCAGC CAGCCACGAC CGAGGCGGTC ACAAACACGA } \\
\text { TCGCAAGAGG GCCGTCAGC CAGCCACGAC CGAGGCGCTC ACAAACACGA }\end{array}$ \\
\hline $\begin{array}{l}\text { Assemblage C } \\
\text { Dog No2 }\end{array}$ & $\begin{array}{l}\text { AGCTCGTCGA G } \\
\text { AGCTCGTGA G }\end{array}$ \\
\hline
\end{tabular}

D) Assemblage D- Accession number AY545647.1

$\cdots|\cdots|_{10} \cdots|\cdots|_{20} \cdots|\cdots|_{30} \cdots|\cdots|_{40} \cdots|\cdots|_{50}$

Assemblage D GAACGAGATC GAGGTCCGCC GCGTCGACGA TGACACGCGT GTCAAGATGA Dog No3 GAACGAGATC GAGGTCCGCC GCGTCGACGA TGACACGCGT GTCAAGATGA

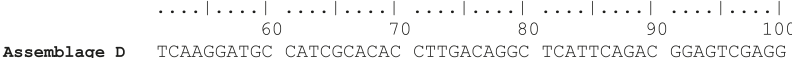
Dog 030 TCAAGGATC CATCGCACAC CTTGACAGGC TCATTCAGAC GGAGTCGAGG Dog No3 TCAAGGATGC CATCGCACAC CTTGACAGGC TCATTCAGAC GGAGTCGAGC

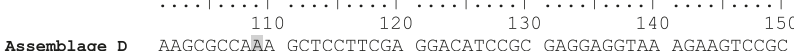
Dog No3 AAGCGCCAGA GCTCCTTCGA GGACATCCGC GAGGAGGTAA AGAAGTCCGC

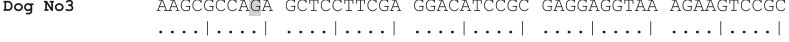
Assemblage D TCACAACATC TATCTCACCA " TCAAGGAGGA CATTGACACA ATGCCCGCAA Dog No3 TGACAACATG TATCTGACGA TCAAGGAGGA GATTGACACA ATGGCCGCAA $\ldots|\ldots| \ldots|\ldots| \ldots|\ldots| \ldots|\ldots| \ldots|\ldots| \ldots|\ldots|$ $\left.\left.\left.\cdots|\cdots|\right|_{210} \cdots|\cdots|{ }_{220} \cdots|\cdots|{ }_{230} \cdots|\cdots|\right|_{240} \cdots|\cdots|\right|_{250}$

Assemblage D ACTTCCGCAA GTCCCTCGCA GAGATGGGCG AGACGCTCAA CAACGTCGAG Dog No3 ACTTCCGCAA GTCCCTCGCA GAGATGGGCG AGACGCTCAA CAACGTCGAG

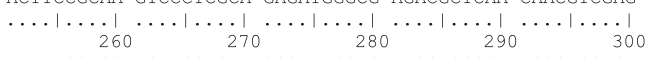
$\begin{array}{lll}\text { Assemblage D } & \text { ACAAACCTCC AGAACCAGAT CGCCATCCAC AACGACGCCA TCGCAGCTCT } \\ \text { Dog No3 } & \text { ACAAACCTCC AGAACCAGAT CGCCATCCAC AACGACGCCA TCGCAGCTCT }\end{array}$

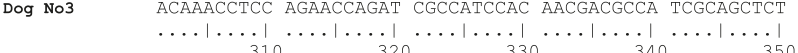
Assemblage D CAGGAAGGAG GCCCTCAAGA GCCTGAACGA CCTTGAGACC GGCATCGCTA $\begin{array}{ll}\text { Assemblage D } & \text { CAGGAAGGAG GCCCTCAAGA GCCTGAACGA CCTTGAGACC GGCATCGCTA } \\ \text { Dog No3 } & \text { CAGGAAGGAG GCCCTCAAGA GCCTGAACGA CCTTGAGACC GGCATCGCTA }\end{array}$

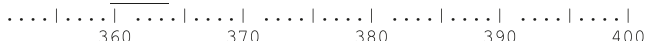

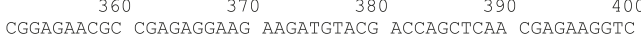
CGGAGAACGC CGAGAGGAAG AAGATGTACG ACCAGCTCAA CGAGAAGGTC $\ldots|\ldots| \ldots|\ldots| \ldots|\ldots| \ldots|\ldots| \ldots|\ldots| \ldots|\ldots|$ GCAGAGGGAT TCGCCCGTAT TTCCGCTGCC ATCGAGAagG AGACGATCGC GCAGAGGGAT TCGCCCGTAT TTCCGCTGCC ATCGAGAAGG AGACGATCGC

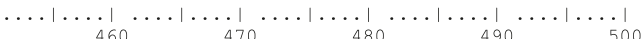

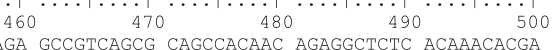
CCGCGAGAGA GCCGTCAGCG CAGCCACAAC AGAGGCTCTC ACAAACACGA

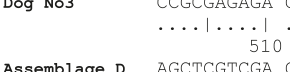

Dog No3 AGCTCGTCGA 


\begin{tabular}{|c|c|}
\hline & E) Assemblage F- Accession number AY647264 \\
\hline & $\cdots|\cdots|_{10} \cdots|\cdots|_{20} \ldots$ \\
\hline Assemblage $\mathrm{F}$ & CGCC GCGTCGACGA CGACACGCGC GTGAAGATGA \\
\hline Cat No2 & 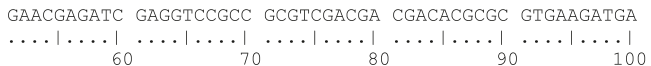 \\
\hline Assemblage $\mathrm{F}$ & TCAAGGACGC CATCGCGCAC CTCGACAGGC TCATCCAGAC GGAGTCGAGG \\
\hline Cat No2 & 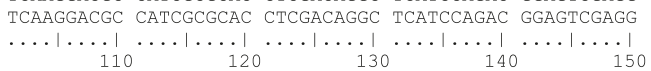 \\
\hline Assemblage $\mathbf{F}$ & AAGCGCCAGG CCTCGTTCGA GGACATCCGC GAGGAGGTCA AGAAGTCCGC \\
\hline Cat No2 & 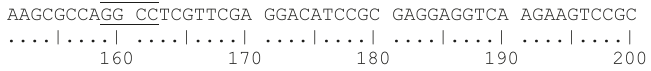 \\
\hline Assemb1 & CGACAACATG TACCTGACGA TCAAGGAGGA GATCGACACC ATGGCAGCCA \\
\hline Cat No2 & $\begin{array}{l}\text { CGACAACATG TACCTGACGA TCAAGGAGGA GATCGACACC ATGGCAGCCA } \\
\cdots \cdots \mid \cdots+1 \\
\cdots\end{array}$ \\
\hline ge $\mathbf{F}$ & ACTTCCGCAA GTCCCTTGCA GAGATGGGCG ACACACTCAA CAATGTTGAG \\
\hline Cat No2 & 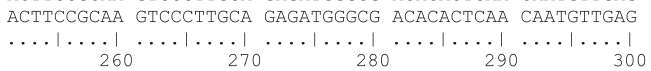 \\
\hline Assembl & ACAAACCTCC AGAACCAGAT CGCCATCCAC AACGACGCCA TCGCGGCCCT \\
\hline Cat No2 & $\begin{array}{l}\text { ACAAACCTCC AGAACCAGAT CGCCATCCAC AACGACGCCA TCGCGGCCCT } \\
\cdots+|\cdots|{ }_{310} \cdots|\cdots|{ }_{320} \cdots|\cdots|{ }_{330} \cdots|\cdots|\end{array}$ \\
\hline Assemb & CAGGAAGGAG GCCCTCAAGA GCCTGAACGA CCTCGAGACG GGCATCGCGA \\
\hline Cat No2 & 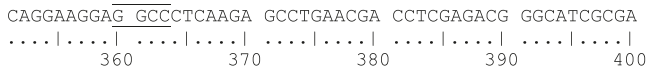 \\
\hline Assemblage $\mathrm{F}$ & CGGAGAACGC AGAGAGGAAG AAGATGTACG ACCAGCTCAA CGAGAAGGTC \\
\hline Cat No2 & 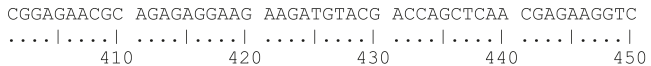 \\
\hline e F & GCAGAGGGCT TCGCCCGCAT CTCCGCCGCC ATCGAGAAGG AGACGATCGC \\
\hline Cat No2 & 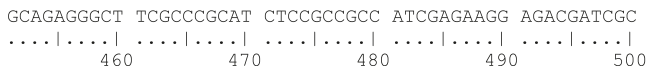 \\
\hline Assemblage $\mathrm{F}$ & CCGCGAGAGG GCCGTCAGCG CCGCCACGAC AGAGGCGCTC ACAAACACGA \\
\hline Cat No2 & 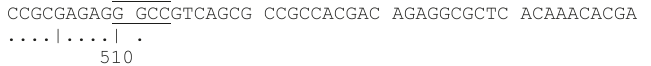 \\
\hline sembla & AGCTCGTCGA G \\
\hline Cat No2 & AGCTCGTCGA G \\
\hline
\end{tabular}
A) The sense strand comparison with assemblage C- Accession
number $\mathbf{A Y 5 4 5 6 4 6}$

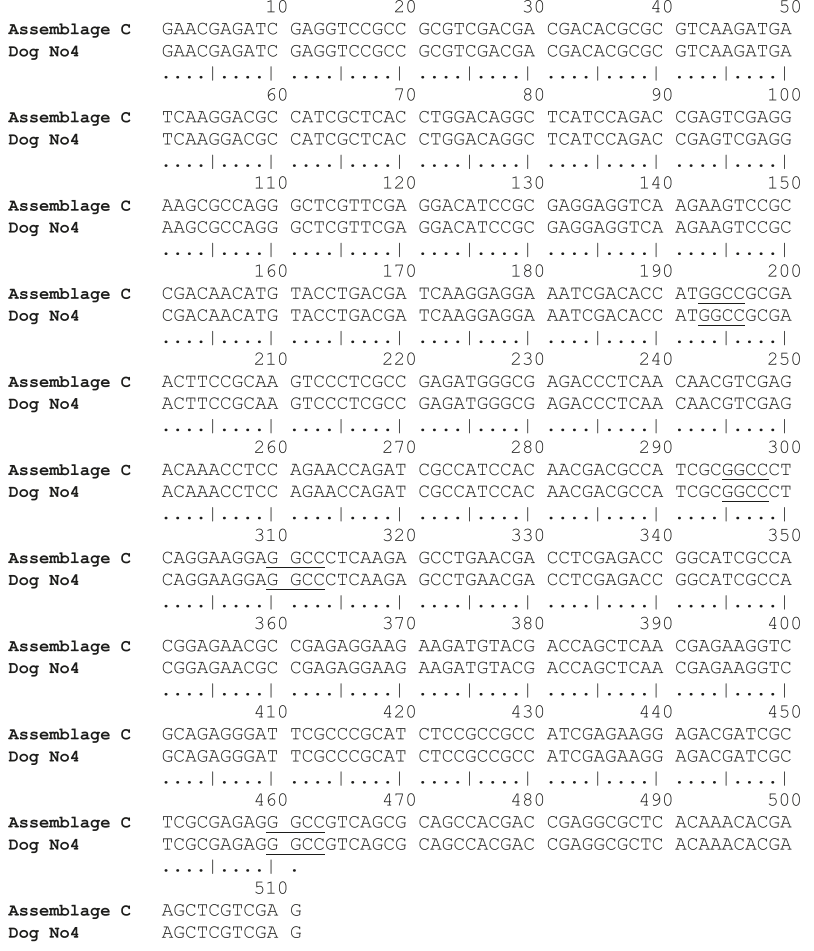

B) The antisense strand comparison with assemblage $\mathrm{C}$

(lack of cut place in 461bp)- Accession number AY545646

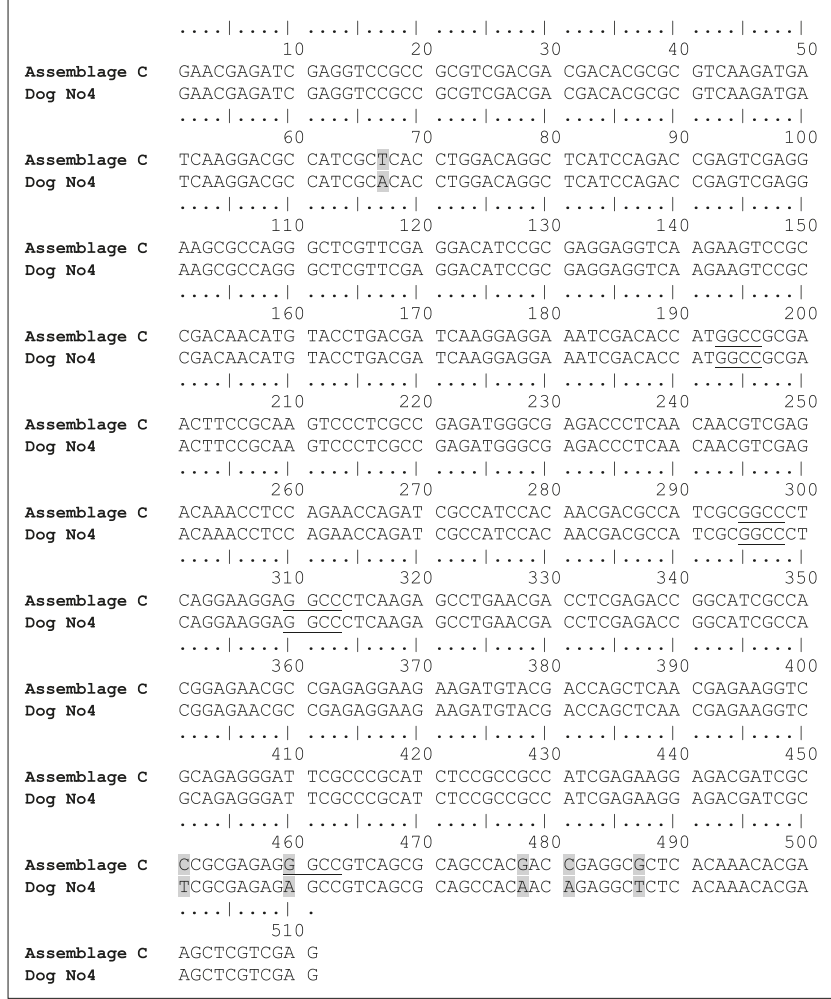

C) The antisense strand comparison with assemblage D (additional cut place in 296bp)- Accession number AY545647.1 $\begin{array}{ll}\text { Dog No4 } & \text { GAACGAGATC GAGGTCCGCC GCGTCGACGA TGACACGCGT GTCAAGATGA } \\ \text { D. } & \end{array}$ $\ldots|\ldots|+\ldots|\ldots| \ldots+\ldots|\ldots|$ Assemblage D TCAAGGATGC CATCGCACAC CTTGACAGGC TCATTCAGAC GGAGTCGAGG Assemblage D
Dog No4 TCAAGGACGC CATCGCACAC CTGGACAGGC TCATCAGAC CGAGTCGAGG

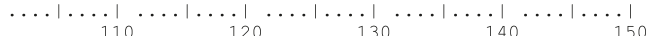
Assemblage D AAGCGCCAAA GCTCCTTCGA GGACATCCGC GAGGAGGTAA AGAAGTCCGC $\begin{array}{ll}\text { Assemblage D } & \text { AAGCGCCAAA GCTCCTTCGA GGACATCCGC GAGGAGGTAA AGAAGICCGC } \\ \text { Dog No4 } & \text { AAGCGCCAGG GCTCGTTCGA GGACATCCGC GAGGAGGTCA AGAAGTCCGC }\end{array}$

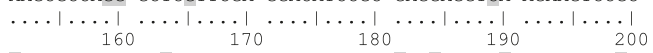
$\begin{array}{ll}\text { Assemblage D } & \text { TGACAACATG TATCTGACGA TCAAGGAGGA GATTGACACA ATGGCCGCAA } \\ \text { Dog No4 } & \text { GGACAACATG TACCTGACGA TCAAGGAGGA AATCGACACC ATGGCCGCGA }\end{array}$

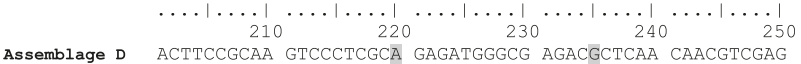
$\begin{array}{ll}\text { Assemblage D } & \text { ACTTCCGCAA GTCCCTCGCA GAGATGGGCG AGACGCTCAA CAACGTCGAG } \\ \text { Dog No4 } & \text { ACTTCCGCAA GTCCCTCGCC GAGATGGGCG AGACCCTCAA CAACGTCGAG }\end{array}$

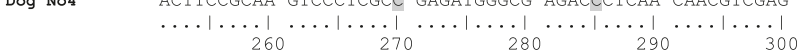
$\begin{array}{lll}\text { Assemblage D } & \text { ACAAACCTCC AGAACCAGAT CGCCATCCAC AACGACGCCA TCGCAGCTCT } \\ \text { Dog No4 } & \text { ACAAACCTCC AGAACCAGAT CGCCATCCAC AACGACGCCA TCGCGGCCCT }\end{array}$ Dog No4 ACAAACCTCC AGAACCAGAT CGCCATCCAC AACGACGCCA TCGCGGCCCT

$\ldots$

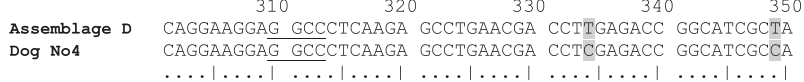

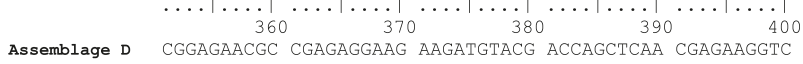
Dog No4 D CGGAGAACGC CGAGAGGAAG AAGATGTACG ACCAGCTCAA CGAGAAGGTC

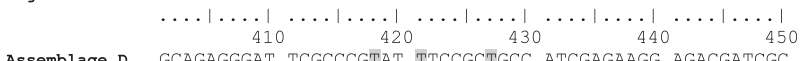

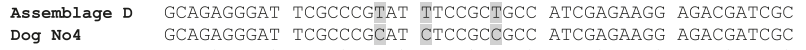

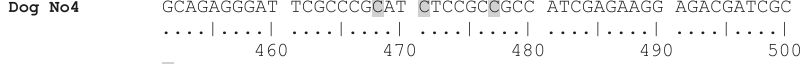
Assemblage D CCGCGAGAGA GCCGTCAGCG CAGCCACAAC AGAGGCTCTC ACAAACACGA Dog No4 TCGCGAGAGA GCCGTCAGCG CAGCCACAAC AGAGGCTCTC ACAAACACGA $\cdots|\cdots|+\mid$

Assemblage D AGCTCGTCGA

Figure 2 A-C. Comparison of G. duodenalis sequencing product from a dog with the pattern of assemblages $C$ and D. Alignment based on maximum similarity of individual assemblages of $G$. duodenalis in the classification of Lalle et al. 2005. The underlined zone indicates a cut place of restrictive enzyme. The grey zone indicates differences in DNA sequence (no consensus at this position) 


\section{DISCUSSION}

Giardia duodenalis occurs in dogs and cats all over the world and is one of the major parasites responsible for the symptoms of the gastrointestinal tract. Extensiveness of the invasion is varied and depends on the geographical location, different hygiene conditions in the region, as well as different diagnostic methods usage. Because the diagnosis of Giardia invasion based on classical methods (faecal flotation or faecal smear) is problematic, commercial immunoassays are therefore increasingly used to detect the presence of parasite protein secretion in animal faeces. Giardiasis is a very common disease and invasion prevalence ranges from $5-80 \%$, depending on the age of the animals. The highest prevalence (46-50\%) is observed in young dogs less than one year old, and in animals with diarrhea $[10,11]$. In Poland in 2001-2006, the prevalence of Giardia invasion in dogs in Poznan was about $10 \%$, in Warsaw it ranged from $9 \%$ to over $50 \%$, in Lublin about 53\%, in Pulawy - $10 \%$ and Gdansk over $16 \%$. Significant differences in prevalence values resulted from the different number of animals in age groups and differences of their clinical condition [12]. In the presented study, the prevalence of G. duodenalis in dogs was $21.1 \%$ and in cats - $15.1 \%$. The use of enzyme immunoassay test for detecting soluble Giardia antigens in faeces increases the probability of invasion detection. Studies conducted in Europe (especially in countries such as the UK, Spain, Netherlands, Italy, Germany, Belgium) using the SNAP Giardia Test (IDEXX Laboratories) showed the presence of G. duodenalis invasion in approximately $25 \%$ of dogs and $20 \%$ of cats with gastrointestinal signs [11]. In the USA, the prevalence of $G$. duodenalis invasion in animals with symptoms of the gastrointestinal tract was $15.6 \%$ in dogs and $10.8 \%$ in cats [13]. Similar results were obtained in Canada, where the prevalence was $13 \%$ in dogs and $4.1 \%$ in cats, and in Japan, where the prevalence of Giardia invasion in 1997-2007 in pet dogs remained unchanged at the level of about $15 \%$ [14]. In Brazil, the percentage of infected dogs was 16.9\% [15]. Usually, for the molecular identification of G. duodenalis, PCR and its modifications (nested-PCR, semi-nested PCR, PCR-RFLP, real-time PCR) or Giardia DNA hybridization with a molecular probes (microarray technique and FISH technique) are used. Analysis of the genetic material is helpful in answering the question whether Giardia detected in a dog or cat can be a source of infection for humans. The most preferred is amplification of the $\beta$-Giardin encoding gene [9]. Giardins are specific structural proteins with a mass of 29-38 kDa, unique for this protozoan [16]. The integral part of each trofozoit sucker are $\alpha$ - and $\beta$-giardins, while on the surface of $G$. duodenalis cell membrane, a further 10 specific proteins are identified. Genetic research conducted in Poland revealed in affected dogs an occurrence of assemblages A-1, C and $\mathrm{D}$ in Warsaw, and only $\mathrm{C}$ and $\mathrm{D}$ assemblages in Poznan $[17,18]$. Molecular analysis of isolates obtained from cats in Warsaw showed the presence of assemblages A, B and D [19]. Current studies in animals in Wroclaw confirmed the presence of specific assemblages $C, D$, mixed assemblages of $\mathrm{C}$ and $\mathrm{D}$, and zoonotic assemblage $\mathrm{B}$ in stool samples derived from dogs. In cats, the presence of assemblage $F$ was revealed, but the zoonotic assemblage A was also detected. The study of 55 dogs in Germany, with no apparent clinical signs of disease, revealed the presence of genotype A in 60\% of tested animals, mixed infections with assemblages $\mathrm{A}$ and
$\mathrm{C}$ in $27.3 \%$, while the individual assemblages $\mathrm{C}$ and $\mathrm{D}$ were rarely recorded, respectively, in $9.1 \%$ and $3.6 \%$ of dogs.

In dogs in Spain, the most commonly circulating assemblages were B, D, followed by C, A, E and F [20]. The study not only confirmed the high prevalence of $G$. duodenalis among dogs with no clinical signs of disease, but also showed that zoonotic assemblage $\mathrm{A}$ is common in urban pet dogs, and even more common than assemblages typical for dogs. Although dogs owners' stools samples were not studied, the results indicate that a large proportion of urban dogs infected with zoonotic genotypes of Giardia are a reservoir for human invasion [21].

As in the presented study, the results of genotyping of Giardia isolates present in dogs in different regions of the world indicate that in these animals specific assemblages $C$ and D usually predominate. Such results were obtained in dogs in Hungary, Brazil and Australia [22, 15, 23]. Similarly, non-zoonotic assemblages were identified more often in dogs in Italy (C and D), and assemblage A or mixed-induced invasion with zoonotic and non-zoonotic assemblages occurred only in individual animals [9]. Also in Australia, zoonotic assemblages $\mathrm{A}$ and $\mathrm{B}$ have been found only in individual animals, which indicated that dogs and cats are not a significant reservoir of invasive isolates of Giardia for humans [23]. In turn, other researchers from Germany, Japan and Thailand detected zoonotic assemblage A of G. duodenalis in dogs more often than assemblages $C$ and $D$ that are specific for canines [24, 25]. Higher prevalence of zoonotic Giardia assemblages were found in dogs that were kept singly than in dogs kept in groups [22]. This confirms earlier suggestions that individual dogs bred in house are infected by Giardia from the owners or householder, while the dogs kept in high density are infected with parasite assemblages specific for canids.

Although most of the invasion found in cats is caused by non-pathogenic to humans assemblages $\mathrm{D}$ and $\mathrm{F}$, it has been shown that these animals are also a potential source of zoonotic $G$. duodenalis assemblages [5, 9, 22, 23]. Research carried out in Poland on cats from Warsaw confirmed the presence of assemblages $\mathrm{A}$ and $\mathrm{B}$, next to assemblages $\mathrm{D}$ specific for cats [19]. Also, the results of the presented study, in which apart from assemblage F specific for cats, the zoonotic assemblage A was also found, indicating a potential threat to human health.

The issue that in 5 positive samples tested with the SNAP Giardia Test (IDEXX Laboratories) nested PCR failed can be explained by insufficient numbers of parasite's DNA for amplification [26].

\section{CONCLUSIONS}

The results confirm the presence of assemblages $C$ and $D$ in dogs, and assemblage $\mathrm{F}$ in cats in the vicinity of Wroclaw. Stating the presence of assemblage A and B suggests that infected pets can pose a threat to human health. This study describes for the first time the presence of mixed infections within host-specific C and D genotypes in dogs in Poland.

\section{Acknowledgements}

The study was supported by statutory research and development activity funds assigned to the Faculty of Veterinary Medicine, University of Environmental and Life Sciences, Wroclaw, Poland. 


\section{REFERENCES}

1. Ortega YR, Adam RD. Giardia: overview and update. Clin Infect Dis. 1997; 25: 545-549.

2. Itoh N, Muraoka N, Saeki H, Aoki M, Itagaki T. Prevalence of Giardia intestinalis infection in dogs of breeding kennels in Japan. J Vet Med Sci. 2005; 67: 717-718.

3. Xiao L, Fayer R. Molecular characterisation of species and genotypes of Cryptosporidium and Giardia and assessment of zoonotic transmission. Int J Parasitol. 2008; 38: 1239-1255.

4. Dryden MW, Payne PA, Smith V. Accurate diagnosis of Giardia spp. and proper fecal examination procedures. Vet Ther. 2006; 7: 4-14.

5. Monis PT, Andrews RH, Mayrhofer G, Ey PL. Molecular systematics of the parasitic protozoa Giardia intestinalis. Mol Biol Evol. 1999;16: 1135-1144.

6. Caccio SM, Thompson RCA, McLaughlin J, Smith HV. Unraveling Cryptosporidium and Giardia epidemiology. Trends Parasitol. 2005; 21: 430-437.

7. Traub RJ, Monis PT, Robertson I, Irwin P, Mencke N, Thompson RC. Epidemiological and molecular evidence supports the zoonotic transmission of Giardia among humans and dogs living in the same community. Parasitology. 2004; 128: 253-262.

8. Read CM, Monis PT, Thompson RC. Discrimination of all genotypes of Giardia duodenalis at the glutamate dehydrogenase locus using PCR-RFLP. Infect Genet Evol. 2004; 4; 125-130.

9. Lalle M, Pozio E, Capelli G, Bruschi F, Crotti D, Caccio SM. Genetic heterogeneity at the beta-giardin locusamong human and animal isolates of Giardia duodenalis and identification of potentially zoonotic subgenotypes. Int J Parasitol. 2005: 35: 207-213.

10. Itoh N, Kanai K, Hori Y, Hoshi F, Higuchi S. Prevalence of Giardia intestinalis and other zoonotic intestinal parasites in private household dogs of the Hachinohe area in Aomori prefecture, Japan in 1997, 2002 and 2007. J Vet Sci. 2009; 10: 305-308.

11. Epe C, Rehkter G, Schnieder T, Lorentzen L, Kreienbrock L. Giardia in symptomatic dogs and cats in Europe-results of a European study. Vet Parasitol. 2010; 173: 32-38.

12. Polozowski A, Piekarska J, Pacon J, Zawadzki W, Bednarz-Nabzdyk R, Cislo-Pakuluk A, Stochnij P. Prewalencja inwazji Giardia duodenalis u psów i kotów z terenu Wrocławia i Dolnego Śląska. Magazyn Weterynaryjny. 2011; 2: 168-172.

13. Carlin EP, Bowman DD, Scarlett JM, Garrett J, Lorentzen L. Prevalence of Giardia in symptomatic dogs and cats throughout the United States as determined by the IDEXX SNAP Giardia test. Vet Ther. 2006; 7: 199-206.
14. Olson ME, Leonard NJ, Strout J. Prevalence and diagnosis of Giardia infection in dogs and cats using a fecal antigen test and fecal smear. Can Vet J. 2010; 51: 640-642.

15. Paz e Silva FM, Monobe MM, Lopes RS, Araujo JP Jr. Molecular characterization of Giardia duodenalis in dogs from Brazil. Parasitol Res. 2011; 110: 325-334.

16. Caccio SM, De Giacomo M, Pozio E. Sequence analysis of the betagiardin gene and development of a polymerase chain reaction restriction fragment length polymorphism assay to genotype Giardia duodenalis cysts from human faecal samples. Int J Parasitol. 2002; 32: 1023-1030.

17. Zygner W, Jaros W, Skowrońska M, Bogdanowicz-Kamirska M, Wędrychowicz H. Prevalence of Giardia intestinalis in domestic dogs in Warsaw. Wiad Parazytol. 2006; 52: 311-315.

18. Stolarczyk P, Majewska AC. A survey of the prevalence and genotypes of Giardia duodenalis infecting household and sheltered dogs. Parasitol Res. 2010; 106: 1015-1019.

19. Jaros D. Zygner W, Jaros S, Wędrychowicz H. Detection of Giardia intestinalis assemblages A, B and D in domestic cats from Warsaw, Poland. Pol J Microbiol. 2011; 60: 259-263.

20. Dado D, Montoya A, Blanco MA, Miró G, Saugar JM, Bailo B, Fuentes I.Prevalence and genotypes of Giardia duodenalis from dogs in Spain: possible zoonotic transmission and $\mathrm{p}$ ublic health importance. Parasitol Res. 2012; 111(6): 2419-2422.

21. Leonhard S, Pfister K, Beelitz P, Wielinga C, Thompson R.C. The molecular characterization of Giardia from dogs in southern Germany. Vet Parasitol. 2007; 150: 33-38.

22. Monis PT, Andrews PT, Mayrhofer G, Ey PL. Genetic diversity within the morphological species Giardia intestinalis and its relationship to host origin. Infect Genet Evol. 2003; 3: 29-38.

23. Palmer CS, Traub RJ, Robertson I., Devlin G, Rees R, Thompson RC. Determining the zoonotic significance of Giardia and Cryptosporidium in Australian dogs and cats. Vet Parasitol. 2008; 154: 142-147.

24. Itagaki T, Kinoshita S, Aoki M, Itoh N, Saeki H, Sato N, Uetsuki J, Izumiyama S, Yagita, K,. Endo T. Genotyping of Giardia intestinalis from domestic and wild animals in Japan using glutamate dehydrogenase gene sequencing. Vet Parasitol. 2005; 144: 283-287.

25. Inpankaew T, Traub R, Thompson RC, Sukthana Y. Canine parasitic zoonoses in Bangkok temples. Southeast Asian J Trop Med Public Health. 2007; 38: 247-255.

26. AdamskaM, Leońska-Duniec A, Maciejewska A, Sawczuk M, Skotarczak B. Recovery of DNA of Giardia intestinalis cysts from surface water concentrates measured with PCR and real time PCR. Parasite. 2011; 18: 341-343. 